\title{
LA MEDIACIÓN COMO INTERVENCIÓN INTERDISCIPLINAR: UNA APROXIMACIÓN A LOS ÁMBITOS Y MODELOS DE MEDIACIÓN EN ESPAÑA
}

\author{
MEDIAÇÃO COMO INTERVENÇÃO INTERDISCIPLINAR: UMA \\ ABORDAGEM AOS CAMPOS E MODELOS DE MEDIAÇÃO NA ESPANHA
}

\section{MEDIATION AS INTERDISCIPLINARY INTERVENTION: AN APPROACH TO THE FIELDS AND MEDIATION MODELS IN SPAIN}

\author{
Enrique Pastor Seller ${ }^{1}$
}

\begin{abstract}
RESUMEN
El artículo presenta un análisis teórico, normativo, metodológico y aplicado de la mediación como una intervención interdisciplinar eficaz en la resolución de los conflictos en la realidad relacional contemporánea de España. Presenta las fortalezas sinérgicas y de construcción de ciudadanía que aporta la mediación, el rol de mediador desde una perspectiva de "empowerment" y los diferentes ámbitos, contextos y prácticas en los que implementar la mediación, concretamente ciudadana, intercultural, educativa y sanitaria.
\end{abstract}

Palabras clave: mediación, intervención interdisciplinar, mediador, conflictos, ámbitos de mediación.

\section{RESUMO}

O trabalho apresenta uma análise teórica, a mediação regulamentar, metodológica e aplicada como uma intervenção eficaz interdisciplinar na resolução de conflitos na realidade contemporânea relacional da Espanha. Apresenta as fortalezas sinérgicas e de construção de cidadania que a mediação fornece, o papel de mediador em situação de poder e as diferentes áreas, contextos e práticas onde se pode implementar a mediação cidadã, intercultural, educativa e sanitária.

Palavras-chave: Intervenção. Mediação interdisciplinar. Mediador. Áreas de mediação de conflitos.

\begin{abstract}
The paper presents a theoretical analysis about regulatory and methodological mediation applied as an effective interdisciplinary intervention in the resolution of conflicts in the contemporary relational reality of Spain. The paper presents the synergistic strengths and building citizenship that is provided by mediation, the role of the mediator in power situations and the different areas, contexts and practices in which it is posible to implement civic, intercultural, educational and health mediation.
\end{abstract}

Keywords: Intervention. Mediation. Interdisciplinar. Mediator. Conflict mediation areas.

\footnotetext{
${ }^{1}$ Profesor Titular de Universidad. Doctor en Sociología. Decano de la Facultad de Trabajo Social de la Universidad de Murcia. Investigador Principal del Grupo de Investigación “Trabajo Social y Servicios Sociales” de la Universidad de Murcia. Email: epastor@um.es
} 


\section{Potencialidades de la mediación en la sociedad relacional}

El intenso ritmo de los cambios que acontecen en nuestras sociedades complejas, en el naciente tercer milenio, está dando lugar a profundas transformaciones en el modelo de sociedad, en la que aparecen nuevas configuraciones institucionales y políticas en la reorganización tanto de finalidades, instrumentos, como de los actores que intervienen en el Bienestar de la ciudadanía y que nos conduce al Estado social de cuarta generación denominado relacional. El actual pluralismo del Bienestar conlleva la redefinición de los papeles de los distintos sectores que componen la sociedad: Estado, mercado, entidades de iniciativa social o tercer sector y redes primarias o solidarias (familia y redes informales), un replanteamiento de las transacciones de los actores sociales orientadas a generar liderazgos compartidos (pluralismo participativo).

La ciudadanía exige calidad y eficacia en la prestación de los servicios públicos y participar en la definición y articulación de las políticas públicas que orientan sus ámbitos de convivencia y decisión. De ahí que el reto sea crear condiciones y espacios de implicación ciudadana que generen oportunidades reales y favorables para la deliberación y construcción colectiva de políticas y actuaciones a partir de la conformación de preferencias sólidas e informadas entre la ciudadanía en el complejo universo relacional local (PASTOR, 2009; 2011). Las ciudades son lugares del diálogo y del conflicto, un "espacio" con infraestructura "hard" (estructural/tangible) y dinámica "soft" (relacional). En éste sentido, la mediación puede ser un factor clave y materia prima para desarrollar el capital social y la inteligencia cooperativa, al servir no solo para facilitar la resolución alternativa de conflictos sino también para generar una nueva cultura ciudadana. Para ello la mediación debe vincularse con la complejidad social, coordinar significados, producir innovación, posibilitar la deliberación y generar capacidades y procesos sostenidos:

\section{Aproximación a la mediación en España}

En la vertiente normativa, la primera Ley de Mediación fue la Ley Catalana 1/2001 de 15 de marzo, de mediación familiar, y constituyó el primer esfuerzo normativo específico de mediación. Aunque circunscrita al ámbito de la familia realiza una descripción amplia aplicable a cualquier controversia y regula principios deontológicos, funciones del mediador, proceso de acreditación profesional y de prestación de servicios, etc. Posteriormente se van promulgando leyes de similar contenido en las diferentes comunidades autónomas: concretamente la Ley de Galicia 4/2001, de 31 de mayo, reguladora de la mediación familiar; Ley de la Comunidad Valenciana 7/2001, de 26 de noviembre, reguladora de la mediación familiar; Ley de Castilla la Mancha 4/2005, de 24 de mayo, del servicio social especializado de mediación familiar; Ley de les Illes Balears 18/2006, de 22 de noviembre, de mediación familiar; Ley de la Comunidad de Madrid 1/2007, de 21 de febrero, de mediación familiar; Ley del Principado de Asturias 3/2007, de 23 de marzo, de mediación familiar; Ley de la Comunidad autónoma de Andalucía 1/2009, de 27 de febrero, reguladora de la mediación familiar y la Comunidad autónoma del País Vasco, a través de su Ley 1/2008, de 8 de febrero, de mediación Familiar. Quiero destacar la Ley 15/2009, de 22 de julio, de Mediación en el ámbito privado y, para la mediación en materia de consumo y la Ley 22/2010, de 20 de julio, del Código de consumo de Cataluña.

En el ámbito estatal se destaca la Ley 5/2012, de 6 de julio, de mediación en asuntos civiles y mercantiles $^{2}$, donde se reconocen las ventajas de la mediación ${ }^{3}$, su capacidad para dar soluciones prácticas, efectivas y rentables a determinados conflictos entre partes y ello la configura como una alternativa al proceso judicial o a la vía arbitral, de los que se ha de deslindar con claridad. La mediación está construida en torno a la intervención de un profesional neutral que facilita la resolución del conflicto por las propias partes, de una forma equitativa, permitiendo el mantenimiento de las relaciones subyacentes y conservando el control sobre el final del conflicto. Entre los principios se destacan

\footnotetext{
${ }^{2}$ Publicada en el B.O.E. núm.: 162 de 7 de julio de 2012. Incorpora al Derecho Español la Directiva 2008/52/CE del Parlamento Europeo y del Consejo del 21 de mayo de 2008.

${ }^{3}$ Mediación como medio de solución de controversias, cualquiera que sea su denominación, en que dos o más partes intentan voluntariamente alcanzar por sí mismas un acuerdo con la intervención de un mediador ( $\left.\operatorname{art}^{\circ} .1\right)$.
} 
la: voluntariedad y libre disposición; igualdad de las partes e imparcialidad de los mediadores; neutralidad y cofidencialidad.

El modelo de mediación que inspira la Ley se basa en la voluntariedad y libre decisión de las partes y en la intervención de un mediador, del que se pretende una intervención activa orientada a la solución de la controversia por las propias partes. Se basa en la flexibilidad y respeto a la autonomía de la voluntad de las partes y pretende sentar las bases generales de la mediación como medio para favorecer una alternativa frente a la solución judicial del conflicto. Considera la figura del mediador esencial dado que debe ayudar a encontrar una solución dialogada y voluntariamente querida por las partes.

\section{Mediación como espacio interdisciplinar en una cultura de paz}

La mediación es un espacio transicional de diálogo cooperativo en el que, al menos, dos partes implicadas en una determinada situación conflictiva y una tercera mediadora abordan de manera constructiva los diferentes temas que los protagonistas del conflicto desean tratar (BOLAÑOS, 2007). Es, por tanto, una intervención profesional, de carácter interaccional e interdisciplinar, que incide en las relaciones interpersonales, intergrupales y/o interinstitucionales para ayudar a prevenir y/o resolver un conflicto. La consideración de la mediación desde una perspectiva psicosocial implica, necesariamente, tener en cuenta los factores del contexto y los procesos psicosociales que acontecen en su aplicación, en la que están involucradas las relaciones interpersonales, las emociones, la comunicación verbal y no verbal, los procesos de influencia social, efectos del poder y el status de las partes, el desarrollo normativo, etc.

El crecimiento exponencial de los procesos conflictuales se produce en todos los ámbitos: familiar, legal, empresarial, laboral, cívico, internacional, consumo, etc. La definición de lo que es un episodio social de conflicto tiene, según Redorta (2007), gran trascendencia pues constituye nuestra unidad de medida de cara a la futura intervención interdisciplinar. En éste sentido podemos identificar quince tipos distintos de conflictos que serían objeto de la intervención y se relacionan con: recursos escasos, poder, autoestima, valores, estructurales, identidad, normativos, expectativas, inadaptación, información, intereses, atributivos, relaciones personales, inhibición y legitimación. En cuanto a las causas centrales del conflicto, Moore (1995) identifica cinco: problemas de relaciones entre personas, problemas de información, intereses incompatibles o percibidos como tales, fuerzas estructurales y problemas de valores.

La mediación basada en una "cultura de la paz" como método alternativo se presenta actualmente como una medida eficaz para la resolución de los conflictos, problemas y disputas (MUNUERA, 2012a, p.106) en múltiples contextos y tipos de problemas (VILLAGRASA, 2004). La mediación es el proceso ético de comunicación entre partes en conflicto, que con la ayuda de un mediador imparcial procurará que las personas implicadas en una disputa puedan llegar, por ellas mismas (responsabilidad y autonomía), a establecer un acuerdo que permita recomponer la buena relación y dar por acabado, o al menos mitigado, el conflicto y actuando preventivamente para mejorar las relaciones con los demás. Por consiguiente, al actuar directamente en la mejora de la comunicación y la promoción de la cooperación, ofrece todo un marco de alternativas para la convivencia pacífica y la solución a los conflictos. La mediación es una alternativa a la violencia, a la auto-ayuda o al litigio y se caracteriza por ser un sistema no adversarial, que difiere de los procesos de "counseling", negociación y arbitraje.

En suma, el conflicto es parte natural de la vida, es generador de cambio y no siempre vamos a poder eliminarlo o detenerlo. El desafío radica en transformar el conflicto, aprender a manejar las diferencias. El conflicto es parte inherente del ser humano y, por tanto, de las relaciones interpersonales, intragrupales e interorganizacionales, por lo que el afrontamiento, abordaje y resolución forman parte también de la interacción social y es necesario el concurso de diferentes disciplinas.

\section{Mediación: procesos, modelos y metodología}

En la mediación, el proceso recae en la responsabilidad de los participantes que deben tomar decisiones que influirán en sus vidas y en 
la responsabilidad del mediador para conducir el proceso de manera neutral. Se confiere así autoridad y poder a las personas participantes, orientando interactivamente la solución del problema y el desarrollo de un plan futuro. La meta realista de la mediación es la resolución de las desavenencias o el manejo del conflicto mediante una serie de etapas definidas y la utilización de unas técnicas para lograr los objetivos (FOLBERG; TAYLOR, 1992). Por tanto es una intervención que requiere de un tiempo y un proceso cooperativo creado por las partes en el que deben cumplirse unas determinadas fases. La mediación interviene en la reconciliación o conciliación de las partes en conflicto (con o sin acuerdo) pero también en la prevención y provención del conflicto.

Se pueden distinguir tres modelos/escuelas, principalmente, de entender y practicar la mediación:

1) Modelo o Escuela Tradicional-Lineal (Harvard) de Fisher y Ury (1996), definen la mediación como una negociación colaborativa, asistida por un tercero, con un enfoque teórico que se conoce por la orientación hacia la "resolución de problemas". El conflicto es considerado desde la causalidad lineal y tiene una causa: el desacuerdo. El mediador se ocupará de ayudar a las partes a centrarse en los intereses y salir de las posiciones y se apoya en técnicas de negociación.

2) Modelo o Escuela Transformativa de Bush y Folger (2006), se centra en la mejorara o transformación de las relaciones humanos mediante el establecimiento de un acuerdo y los esfuerzos se orientan para tratar de conseguir cambiar los procesos de relación, aumentar el poder y el reconocimiento de las partes. El conflicto es considerado como algo positivo que posibilita el cambio personal y social, una oportunidad para el crecimiento.

3) Modelo Circular-Narrativo de Coob (1994), pone el énfasis en la comunicación en las categorías de narrativa-circular e historia. Se denomina circular al considerar la comunicación como causalidad y narrativa. Entiende el conflicto asociado al antagonismo en las relaciones humanas, como una realidad interna, presente y continuada en las personas, de etiología circular. Parte de una concepción donde se facilita que las personas manejen el conflicto a base de ayudar a las partes para que puedan cambiar sus historias y puntos de vista acerca de su vivencia de la situación de conflicto.

Otros autores, como Soleto y Otero (2007), incluyen un cuarto denominado Modelo Tópico, que no excluye los anteriores y se centra en la discusión que se produce entre las partes en conflicto por el bien o el derecho que ambas se disputan, siendo necesario localizar -el sentido común y trabajar sobre los referentes de lo justo y lo injusto del conflicto. Otras aplicaciones novedosas son la mediación multiparte (RODRIGUEZ, 2005) y la apreciativa (AMAT; BARREIRO; TATTER, 2006).

La mediación, como metodología de intervención, incluye la acción mediadora entre las distintas actividades que se implementan en la práctica para la resolución del problema y mejorar la calidad de vida. Intenta cambiar, de entrada, los comportamientos de las personas en una situación de conflicto y para poder hacerlo, según el paradigma anidado (DUGAN, 1996), es preciso identificar el origen de las situaciones de conflicto y el nivel de respuesta en el que se está trabajando, más allá de las partes que están en disputa: primer nivel, de problemas concretos; segundo, de problemas vinculados al patrón de interacción y/o de relación entre las partes; tercer nivel, de conflictos sistémicos y subsistémicos.

Por todo lo anterior, la mediación es una intervención interdisciplinar que debe ser planteada en razón de: las causas originarias del conflicto y su nivel de respuesta; si los objetivos se dirigen a manejar mejor una situación de crisis; si se desea transformar y/o cambiar una situación de crisis; si se desea prevenir la emergencia de nuevo de una situación de crisis y si se desean cambiar las estructuras y/o las relaciones sociales.

\section{Rol del mediador orientado al empowerment}

\section{El mediador es}

La persona u órgano que facilita el acuerdo, asiste a las partes para acercarla, crea un ambiente propicio para su comunicación y asegura que las partes actúan con igualdad de armas, en igualdad de condiciones, reequilibrando si ello es necesario. (CASANOVAS, MAGRE; LAUROBA, 2011, p.158). 
Como afirma Moore (1995), el mediador desempeña el rol de facilitador de la comunicación entre las partes y no es árbitro ni juez, ya que no es él quien resuelve en definitiva, sino que lo hacen las partes mismas. Los mediadores son los defensores de un proceso equitativo y no de un determinado resultado, carecen de poder de decisión autorizado, esto es lo que los distingue, básicamente, del juez o del árbitro. Para Grover, Grosch y Olczak (1996), cuando la comunicación entre las partes se vuelve tensa o inexistente, aumenta la intensidad del conflicto, por ello el mediador busca mejorar la capacidad de las partes para comunicarse y explorar actitudes y posiciones mutuas.

Con respecto a la comunicación, para Moore (1995), la función del mediador es la de facilitador de la comunicación entre las partes para que no se produzcan interferencias que dificulten el entendimiento en las comunicaciones, posibilitando de esta manera que lo que cada parte exprese sea interpretado por la otra parte en su esencia, libre de las interferencias producidas por resentimientos, desconfianza y/o resquemores. Siguiendo la línea de este autor, la mediación es flexible puesto que el proceso no sigue un orden predeterminado y que no está sometido a las reglas legales. Es voluntario porque las personas ingresan al mismo por propia decisión, ellas mismas determinan cual es la información que transmiten y cual ocultan, deciden si llegan o no a un acuerdo y se retiran cuando ellas así lo estimen, en el mismo instante en que dejen de sentirse cómodos. Por lo tanto, los acuerdos que se logran son muy eficaces, teniendo una mayor probabilidad de éxito por la carga emocional que posee algo que decidimos en conjunto. Contempla los intereses de ambas partes, es un proceso de triunfo, todos ganan, el énfasis no está puesto en quien gana o pierde sino en establecer las necesidades de los participantes y esto es precisamente lo que lo hace eficaz. Atendiendo a la confidencialidad del proceso, el mediador no podrá reproducir nada de lo que en este procedimiento se diga, ni ser citado como testigo, pues lo ampara el secreto profesional. El mediador, como señalan Pastor e Iglesias (2011), trabaja para ayudar a las partes a examinar el futuro y sus intereses o necesidades y a negociar el intercambio de promesas y relaciones que serán mutuamente satisfactorias y se ajustarán a las normas de equidad de dichas partes.

La mediación logra a través del proceso creativo encontrar un mayor número de soluciones que las que los participantes perciben desde sus posiciones iniciales/actuales, activando su capacidad de negociación en base a sus verdaderos intereses y necesidades, facilitando acuerdos en el que ambas partes se sientan ganadoras. De esa forma, los que intervienen no sienten que la decisión está fuera, sino que comienzan a percibirla desde la perspectiva del otro y con ello, lograran ver la implicación, la responsabilidad y la colaboración. Esta recuperación de la responsabilidad se vincula como el "empowerment" y es una de las características de la mediación que la convierte en un proceso útil para los individuos, grupos y organizaciones. El "empowerment" supone transferir las capacidades, competencias y habilidades para que las personas, grupos $\mathrm{y}$ organizaciones puedan determinar $\mathrm{y}$ focalizar las causas del conflicto y sean protagonistas del proceso y el resultado final de la mediación. En mediación, como señala Munuera (2012 b), es una estrategia que utiliza el enfoque apreciativo (EA) para la transformación, tanto de organizaciones como de personas, a través del cambio en la forma de comunicarse, pasando, de un lenguaje deficitario cargado de quejas, a un lenguaje apreciativo. Este enfoque trabaja desde un ciclo dinámico de cuatro procesos llamados 4D's: descubrimiento, deseo, diseño y destino; cada uno de los cuales está dirigido a alcanzar los objetivos que el sistema (persona $u$ organización) desea.

\section{Ámbitos y contextos de intervención de la mediación}

Las variaciones metodológicas entre los diferentes ámbitos de la mediación tendrán lugar en el procedimiento, herramientas y técnicas empleadas por el mediador, pero la base filosófica es similar.

En relación a los ámbitos de intervención de la mediación son amplios y diversos: empresarial, laboral, consumo, ciudadana y comunitaria, familiar, escolar, salud, penal para jóvenes y adultos $\mathrm{y}$ medioambiental. A continuación nos centraremos en los ámbitos de intervención de la mediación vinculados con contextos de protección de bienestar 
social, concretamente comunitaria, intercultural, educativa y sanitaria.

\section{Mediación ciudadana, comunitaria e intercultural}

Se define a partir de la dualidad de tener un carácter transversal y, al mismo tiempo, un campo de actuación propio, fruto de la problemática social existente y de la estructura política local. El gobierno local recibe las quejas ciudadanas sobre las numerosas cuestiones que afectan a su convivencia y que carecen de una respuesta definida y operativa; de ahí su implantación desde las administraciones locales. Como señalan Munné, Prokopljevic, Llorens et al. (2011), los conflictos propios de éste ámbito pueden dividirse en dos niveles: privado y público. Al primer nivel le corresponden los de convivencia entre vecinos en espacios privados $y$, al segundo, los de convivencia en un entorno público. Entre los sectores de intervención destacamos: problemas de vecindad, ruido y contaminación acústica, alquileres y vivienda, actos incívicos, etc. Se manifiesta a partir de tres objetivos: entender el conflicto como motor de cambio; promover la autonomía de las personas a través del empoderamiento del individuo y/o grupo, el reconocimiento del otro y favorecer la responsabilidad ciudadana y los vínculos sociales. La responsabilidad ciudadana y la creación de los vínculos sociales, objetivos de la mediación ciudadana y comunitaria, han sido los dos conceptos que han potenciado la creación y el desarrollo de la prácticas y experiencias de mediación en el ámbito local.

Respecto a la mediación intercultural, existen, fundamentalmente, dos visiones; una que concibe a ésta igual que la mediación ciudadana y comunitaria con la particularidad en la diferencia de la cultura de al menos una de las partes confrontadas y otra, la concibe de manera específica como facilitación de la comunicación entre partes de diferentes culturas.

\section{Mediación en el ámbito escolar}

Lamediaciónen elámbito escolar, comoseñalan Carrasco, Villá, Ponferrada y Casañas (2011, p. 501503 ), debe considerare de forma general como una estrategia de resolución dialogada y colaborativa de conflictos que, a diferencia de su aplicación en otros ámbitos, tiene también un carácter de intervención educativa intencional. Esto significa que, si bien todo el proceso de mediación en cualquier ámbito conlleva el aprendizaje de nuevos planteamientos de resolución de conflictos, la mediación en este ámbito es doblemente educativa. Por un lado, se produce una fuerte identificación entre el ámbito institucional donde puede surgir o puede hacerse evidente el conflicto - el escolar y la actividad que allí se desarrolla - la educativa. Por el otro, predomina el énfasis en la mediación escolar como una estrategia principalmente aplicada a los conflictos entre iguales $y$, por tanto, como un conjunto de elementos que deben ser aprendidos por parte del alumnado. Pero la filosofía desde la cual se impulsa la mediación en el ámbito escolar no es solo ni principalmente una cuestión de aprendizaje para el alumnado, tiene implicaciones para la transformación de toda la cultura escolar tradicional y de sus respuestas frente a los conflictos. La orientación pedagógica específica de la mediación escolar corresponde sobre todo a las fases previas, a formación en valores y educación en una cultura no violenta y también entre adultos. Por consiguiente, es una herramienta de resolución de conflictos en el ámbito escolar, con efectos organizativos y económicos, dado que no está limitada a los miembros de la comunidad educativa.

\section{Mediación en el ámbito de la salud}

La mediación en el ámbito de la salud es una metodología alternativa para resolver disputas en el contexto sanitario a través de la figura profesional o de servicio del mediador. Esta mediación, como señalan Armadans et al. (2011, p. 585-586) permite la gestión del cambio y el acompañamiento de las personas en los procesos de prevención, resolución, transformación o contención de las situaciones de conflicto. Una nueva manera de regular socialmente la intervención en la resolución de los conflictos, en la comunicación, en la educación y en la seguridad. También puede formar parte de los nuevos sistemas para gestionar los conflictos entre profesionales y usuarios de los hospitales y centros sanitarios, contribuyendo a una mejor gestión de los cambios, utilizando el diálogo como eje central de las intervenciones en la relación clínica. Como 
señala Lederach (1989) es importante tratar de construir espacios de paz en la vida cotidiana de una organización con el apoyo de procesos de diálogo basados en la cultura dela paz. Y, en este sentido, la mediación se podría establecer como una nueva metodología profesional vinculada a una nueva manera de vivir las relaciones personales en el seno del entorno laboral en el ámbito sanitario.

\section{Conclusiones}

La mediación, es una intervención social centrada en la gestión y resolución de conflictos que dispone de un marco teórico, normativo, principios, proceso, técnicas y características singulares $\mathrm{y}$ propias construidas a partir del enriquecimiento interdisciplinar y la sistematización de sus prácticas.

El crecimiento exponencial de los procesos conflictuales se produce en todos los ámbitos: familiar, legal, empresarial, laboral, cívico, internacional, consumo, etc. dado que el conflicto es parte inherente del ser humano y, por tanto, de las relaciones interpersonales, intragrupales e interorganizacionales, por lo que el afrontamiento, abordaje y resolución requieren de una intervención interdisciplinar.

En la actual sociedad del conocimiento disminuye la disposición a aceptar las decisiones adoptadas de manera jerárquica, se demandan nuevas formas de adopción de decisiones y de comunicación que incidan en una mayor implicación, capacidad y compromiso de la ciudadanía tanto en la definición de problemas como en la gestión de sus soluciones alternativas. La mediación nos aporta elementos sustantivos para emprender una nueva forma de concebir la realidad social e implementar intervenciones desde la autonomía de sus actores. Es necesario disponer de una ciudadanía en mayúsculas y la mediación puede ser un factor clave y materia prima para desarrollar el capital social y la inteligencia cooperativa, al servir no solo para facilitar la resolución alternativa de conflictos sino también para generar una nueva cultura ciudadana.

La creciente intervención profesional en la resolución de los conflictos, el impulso del desarrollo normativo en el ámbito nacional y autonómico y el incremento de los servicios de mediación en diferentes sistemas de protección, entre otros, refuerzan los argumentos de quienes sostienen que la mediación es interdisciplinar, con un cuerpo teórico y práctico, así como principios y reglas básicas. Las prácticas y resultados de la mediación constatan ser eficaces para la resolución de los conflictos, problemas y disputas en múltiples contextos y tipos de problemas.

\section{Referencias}

AMAT, P.; BARREIRO, A.; TATTER, M. Hacia un nuevo paradigma: la mediación apreciativa. In: Jornadas de diálogos apreciativos: el socio-construccionismo en acción. Oñati, 10-11 abril, 2006.

ARMADANS, M., ANEAS, E., MONGE E IGUAL La mediación en el ámbito de la salud. En: CASANOVAS, P., MAGRE, J.; LAUROBA, M. E. (Dirs.). Libro blanco de la mediación en Cataluña. Barcelona: Generalitat de Catalunya, 2011. p. 579-648.

BOLAÑOS, I. Mediación transicional. Revista Portularia, v. 7, n. 1-2, p. 61-74, 2007.

BUSH, R.; FOLGER, J. P. La promesa de la mediación: cómo afrontar el conflicto a través del fortalecimiento propio y el reconocimiento de los otros. Buenos Aires: Granica. 2006.

CARRASCO, V., PONFERRADA y CASAÑAS La mediación en el ámbito escolar. En: CASANOVAS, P., MAGRE, J.; LAUROBA, M. E. (Dirs.). Libro Blanco de la Mediación en Cataluña. Barcelona: p. 497-578, 2011.

CASANOVAS, P., MAGRE, J.; LAUROBA, M. E. (Dirs.). Libro Blanco de la Mediación en Cataluña. Barcelona. 2011.

COOB, S. A Narrative perspective on mediation. En: FOLGER Y JONEX, T. (Comp.). New directions in mediation: communication, research and perspectives. Londres: Sabe Publications, p.48-63, 1994.

DUGAN, M. A nested theory of conflict. Women in Leadership, v. 1, n. 1, p. 9-20, 1996.

FISHER, R.; URY, W. Obtenga el sí: el arte de negociar sin ceder. Madrid: Ediciones Gestión, 1996.

FOLGER, J.; TAYLOR, A. Mediación: resolución de conflictos sin litigio. México: Limusa, 1992.

GROVER, K; GROSCH, J. K.; OLCZAK, P. V. La mediación y sus contextos de aplicación. Barcelona: Paidós, 1996.

LEDERACH, J. P. Elementos para la resolución del conflicto. México: Servicio de Paz y Justicia, 1989.

MOORE, C. El proceso de mediación: métodos prácticos para la resolución de conflictos. Barcelona: Granica, 1995. 
MUNNÉ, M.; PROKOLIEVIC, L. 1. et al. Mediación ciudadana y comunitaria. En: CASANOVAS, P., MAGRE, J.; LAUROBA, M. E. (Dirs.). Libro Blanco de la Mediación en Cataluña. Barcelona: Generalitat de Catalunya, p. 375432, 2011.

MUNUERA, P. J. M. Haynes, Perlman, Chandler y otros autoresinternacionales en el recorrido de mediación y Trabajo Social. Revista Portularia, v. 12, n.2, p. 97-108, 2012. (a)

Conflicto, Mediación y Trabajo Social. En: FOMBUENA, J. Trabajo Social y sus instrumentos: elementos para una interpretación a piacere. Valencia: Nau Llibres, 2012 (b). p. 171-190.

PASTOR, E. Participación ciudadana y gestión de políticas sociales municipales. Murcia: Editum, 2009.

Proximity social policies government: quality and democratic improvement analysis of local council of well being (Murcia, Spain). Revista de Administração Pública, v. 45, n. 2, p. 377-399, 2011.

PASTOR, E.; IGLESIAS, E. La mediación intrajudicial como método de resolución de conflictos en el seno familiar. Entramado, v. 7, n. 2, p. 72-87, 2011.

REDORTA, J. Entender el conflicto: la forma como herramienta. Barcelona: Paidós.2007.

RODRIGUEZ, C. Innovaciones de la mediación: la mediación multiparte. Aplicaciones de la mediación al trabajo con grupos. Trabajo Social Hoy, v. 52 n. 1, p. 135145, 2005.

SOLETO, H.; OTERO, M. Mediación y solución de conflictos: habilidades para una necesidad emergente. Madrid: Tecnos, 2007.

VILLAGRASA, C. (Cord.). La mediació: L'alternativa multidisciplinària a la resolució dels conflictes. Barcelona: Universidad de Barcelona y Pòrtic, 2004.

Recebido em: 26 de março de 2013.

Aceito em: 01 de junho de 2013. 